\title{
La publicidad en la Prensa del Movimiento
}

\author{
Carlos de las Heras Pedrosa \\ Universidad de Málaga \\ cheras@uma.es \\ Carmen JAMBRino MALDONADO \\ Universidad de Málaga \\ mcjambrino@uma.es
}

\begin{abstract}
Resumen:
Desde los inicios del Régimen franquista con la Prensa del movimiento se observa en el conjunto de sus publicaciones una marcada ausencia de estructuración del departamento comercial, ello conlleva que el papel desempeñado por la publicidad adquiera a lo largo de este período diferentes connotaciones. La falta de visión empresarial sobre todo en los primeros veinte años supuso un elevado coste que tuvieron que asumir los diferentes diarios de la Cadena. Más tarde, la insostenible situación provoca una normalización comercial y publicitaria que marca la última etapa, la publicidad llega a convertirse en el único recurso aparente para el sustento económico de la Prensa del movimiento.
\end{abstract}

Palabras Clave: Prensa del Movimiento; publicidad; periodismo; historia del periodismo; Franquismo.

\section{Advertising in Newspapers Movement}

\begin{abstract}
:
From the beginning of the Franco regime's Press Movement seen in all its publications structuring a marked absence of commercial department, this implies that the role of advertising purchases over this period different connotations. The lack of entrepreneurial vision especially in the first twenty years was a high cost had to take different day of Chain. Later, the unsustainable situation causes commercial and advertising standards that mark the last stage; advertising gets to become the only apparent recourse for economic sustenance of the Movement Press.
\end{abstract}

Key Words: Movement Press; advertising; journalism; journalism history; Franquismo

\section{Referencia normalizada:}

De las Heras Pedrosa, C. y Jambrino Maldonado, C. (2014): La publicidad en la prensa del movimiento. Historia y Comunicación Social. Vol. 19. Núm. Especial Febrero. Págs. 847-860.

Sumario: 1. Introducción, 2. Estructuración del sector: los agentes publicitarios, 3. Hacia el Rendimiento Económico, 4. La División Comercial, 5. El Anuncio Nacional, 6. Conclusiones, 7. Bibliografía

\section{Introducción}

El presente artículo es un análisis historiográfico de la publicidad en la Prensa del Movimiento. Basándonos en los legajos depositados en el Archivo General de la Administración, en su sección de cultura, hemos reconstruido los diferentes procesos en los que se desarrolló la gestión de la publicidad y su importancia en los periódicos 
de la Cadena. La documentación en poder del Archivo General de la Administración (AGA) sobre la Prensa del Movimiento es cuantiosa y muy valiosa, al ser un organismo público toda la documentación, informes, cartas, etc. que confeccionaba el personal de los distintos periódicos locales era remitida a la central donde eran registradas y archivadas. Gracias a ello, hemos podido ir descubriendo con verdadero detalle cada momento por los que atravesaba las diversas publicaciones. En este artículo hemos profundizado en la publicidad centralizada y en la organización de su departamento comercial en las distintas etapas por la que discurre la dictadura franquista y que se reflejan claramente en las políticas tanto informativas como publicitarias de la Prensa del Movimiento.

Desde los inicios, la publicidad no sólo fue un elemento poco considerado en la Prensa del Movimiento, sino también criticado por restar espacio a la línea propagandística.

De una etapa de pluralismo expresivo y de creciente esplendor publicitario pasamos a una publicidad de carácter mixto entre lo económico y lo político ${ }^{1}$, así, nos encontraremos con anuncios comerciales aclamando las victorias del ejército franquista o la toma de una nueva ciudad, con textos como el siguiente, dirigido a los niños.

"Vuestra alegría infantil es lo mejor que, hoy, podéis ofrecer a la Patria, usando a diario polvos higiénicos Calber"2.

La publicidad al servicio del Estado Franquista era un elemento de segundo orden. El interés primordial de los dirigentes de la Cadena era la educación de los españoles a través de sus periódicos, de ahí que fuese prioritario el espacio de los diarios para propagar los nuevos ideales del Régimen. No importaba que los periódicos no fuesen rentables en el ámbito económico si lo eran en el propagandístico.

La finalidad comercial que encontraban los anunciantes en la publicidad no era asumido por los periódicos de la Cadena. La actividad publicitaria dependería durante muchos años del departamento de administración y de estadística, al igual que las ventas o la distribución. No existía un órgano que se dedicase a la gestión publicitaria en el ámbito local y mucho menos en el nacional.

El espacio reservado a la publicidad en los primeros años de la Cadena no sobrepasaba el 20\%. Existía escasez de papel y era primordial la utilización de los periódicos de la Prensa del Movimiento para educar a la población en el marco general del nuevo Régimen.

Aun manteniendo estos criterios, la presión sobre la publicidad en los diarios de la Cadena no era solamente un tema de espacio. A la publicidad se le exigiría algo más,

1 CIRICI PELLICER, A. (1977). La estética del franquismo, Gustavo Gilli, Barcelona.

2 Anuncio publicado en el Sur de Málaga en conmemoración a la toma de la ciudad por las tropas de Francisco Franco el 8 de febrero de 1937. 
debería ir acorde con los caminos informativos y educadores de la propia prensa, debiendo servir como un elemento más del periódico. Así lo expresa Prados y López ${ }^{3}$

"La misión periodística tiene que ser servida por cuantos con la Prensa se relacionan, incluso por los anunciantes; los cuales, después de todo, no pierden nada con seguir las directrices periodísticas y plegarse al interés de la Prensa, al sentido ético y estético de la misma."

La Prensa se convierte en creativa publicitaria y marca las líneas a seguir de los anuncios que se insertaban en los diarios, exigiendo que el texto de sus anuncios fuera moral, grato, sugestivo y artístico, gracias a estos aspectos se conseguiría, según sus criterios, una mayor eficacia publicitaria.

Otro de los aspectos a tener en cuenta es la afirmación de la relación publicidad-política y la utilización de ésta como orientadora, basándose en su prestigio, su consejo y su fuerza mediática.

Estas líneas de actuación en la creatividad de sus anuncios que debían seguir los anunciantes se podían considerar "impuestas" como se desprende del siguiente párrafo de Prados y López:

La coordinación entre el interés del anunciante y el interés del periódico resulta siempre saludable y beneficiosa para todos. Entre, pues, el anunciante en la disciplina del periodismo y no tendrá que arrepentirse ${ }^{4}$.

La publicidad quedaría relegada a un segundo plano, los editores (dueños y señores de sus periódicos), no solamente influirán con sus artículos en la opinión pública, sino que intentarían, aún censurando, que todo aquel que tenga cierta relación con sus diarios se adecuase a los mismos, bien con una línea colaboracionista, o con la rigurosa censura.

\section{Estructuración del sector: los agentes publicitarios}

Los resultados negativos arrojados por diversas publicaciones, a pesar del sistema de compensaciones con aquellas otras con saldos positivos, marcan el comienzo de una etapa donde la publicidad se convierte en el principal elemento de financiación para la supervivencia de muchos títulos.

Se crearon los servicios administrativos de publicidad y pactaron acuerdos con un nutrido número de publicistas para que les gestionaran y consiguieran anuncios para sus explotaciones. Para convencer a los anunciantes se utilizo la imagen de gran

3 PRADOS Y LÓPEZ, M. (1943) Ética y Estética del periodismo español, Espasa-Calpe, Madrid., p. 119.

4 PRADOS y LÓPEZ, M. (1943) Ética y Estética del periodismo español, Espasa-Calpe, Madrid p. 121. 
empresa con páginas más rentables que las de la competencia. Anuncios al respecto pueden verse en los anuarios de la prensa española de la década de los cincuenta y siguientes. El mayor empuje en este campo se dio en 1947, dentro de las previsiones para el ejercicio siguiente.

En 1955 se publicaron las primeras tarifas de publicidad para las publicaciones dependientes de la Gerencia de Provincias, que era el órgano rector de todos los periódicos pertenecientes a la Delegación Nacional de Prensa y Propaganda. Hasta entonces cada diario aplicaba las tarifas y descuentos que entendía oportuno. Como consecuencia de ello, existían grandes diferencias en el trato publicitario que cada director daba a su periódico.

Como consecuencia de la extensa red de publicaciones que dependían de la Gerencia de Provincias, el considerable número de Agentes de Publicidad que colaboraban con las mismas y la importancia del montante que por su gestión se generaba, se elaboró por parte de dicha Gerencia (sección de publicidad) para el año 1954, unas normas de funcionamiento con el fin de regularizar el funcionamiento de los Agentes de Publicidad recogiendo en ellas cuanto pueda interesar para marcar una pauta a seguir, tanto por las Administraciones de la Cadena de Prensa, como por los Agentes de Publicidad que de ellas dependan.

Hasta ese momento, y por parte de todas las entidades periodísticas, se venía realizando una contratación de carácter privado con cada agente, en las que con más o menos amplitud y diversos porcentajes de comisiones se ajustaban a unas condiciones que pudiéramos llamar generales y que posteriormente sirvieron de base para elaborar las normas.

Al mismo tiempo y según texto de las normas "pretendemos, por medio de estas Normas, dignificar la profesión de Agente de Publicidad y afianzar su situación en nuestra Prensa en mutuo beneficio, puesto que la mejor organización de su trabajo repercutirá en los ingresos que por su gestión hayan de obtenerse" 5 .

Con estas normas la Gerencia pretendería controlar el amplio número de agentes publicitarios que había por todo el país, así como todos los impagados que se iban produciendo. Los agentes podrían incorporarse a la Cadena después de un período de prueba.

El Administrador del diario era la persona encargada de coordinar la gestión publicitaria de dicho diario, admitiendo las órdenes publicitarias que le aportasen los Agentes.

Por lo tanto, el Administrador haciendo funciones de lo que hoy en día es el Director Comercial, asumía la responsabilidad de todo lo concerniente a la publicidad, coordinando y gestionado a su equipo de agentes, determinando los anunciantes que se visitaban, así como las diferentes secciones del periódico, los extraordinarios, etc.,

5 ARCHIVO GENERAL DE LA ADMINISTRACIÓN. Sección Cultura. (1953). Normas sobre agentes de publicidad de la Delegación Nacional. Signatura 182. 
que cada agente debía comercializar, para evitar en la medida de lo posible intrusismo de los Agentes en la publicidad directa o en la de otros Agentes publicitarios.

A los Agentes de Publicidad de un diario se le exigía exclusividad, no pudiendo comercializar la publicidad de otros diarios, revistas, emisoras, etc., que no perteneciesen a la Delegación Nacional, sin previa autorización expresa para ello, que se concedía en todos los casos en que por el ámbito de la capital, forma de trabajo, personal, etc., podía considerarse beneficioso a los intereses de la cadena.

Continuando con la exposición de las Tarifas Publicitarias de las publicaciones pertenecientes a la Gerencia de Provincias, entramos ahora en el concepto de publicidad en Cadena. Hemos podido constatar por los anuncios insertados en los diarios que existía la posibilidad de publicar un anuncio en todos los periódicos de la Prensa del Movimiento, pero estos datos no se reflejan por escrito hasta que se publican las primeras normas de actuación de los agentes y, por supuesto, ahora con la publicación de las tarifas oficiales.

Los descuentos ofrecidos a los anunciantes por insertar sus anuncios en todos los periódicos realmente son insignificantes, el descuento es de tan sólo un $15 \%$ lo que hará una media en la Sección General de 1,20 ptas. $\mathrm{m} / \mathrm{m}$. Estos descuentos son rentables para las inserciones realizadas en Barcelona con un coste de 3,35 ptas. $\mathrm{m} / \mathrm{m}$ y en pocas publicaciones más. Se ha de tener en cuenta que los diarios madrileños no pertenecen a la Gerencia de Provincias, llevando su gestión publicitaria de forma independiente. Cabe pensar que el anunciante que desease salir en todos los periódicos de la Prensa del Movimiento, incluido Madrid, tendrían como mínimo estas mismas condiciones de contratación, aunque como ocurría en años anteriores, dependiendo del cliente se aplicaría la tarifa o bien condiciones especiales.

Otro de los aspectos a considerar a la hora de insertar un anuncio en cadena sería las diferencias existentes en las medidas de las páginas. No existía una medida homologada para todas las publicaciones, pudiendo variar desde $43 \mathrm{~m} / \mathrm{m}$. de ancho de columna para el caso de los diarios Información y Lunes de Alicante, hasta $55 \mathrm{~mm}$ en los casos de Patria, Yugo y Jaén de Granada, Almería y Jaén respectivamente.

\section{Hacia el rendimiento económico}

La falta de interés inicial hacia la publicidad como fuente de ingresos compensada, por la consecución de los fines políticos y propagandísticos llega a su fin. La llegada de los tecnócratas al Estado marca una etapa de aperturismo y una gestión orientada hacia el máximo beneficio en todas publicaciones.

En 1957 se elaboró un nuevo organigrama para la Delegación Nacional, que venía marcado por el Decreto de 20 de julio sobre estructura de la Secretaría General del Movimiento, y por la sustitución de Correa Veglisson como Delegado Nacional, por Álvarez Fueyo. En la redacción del artículo primero de la norma se denota un claro 
carácter técnico, atenuándose las funciones políticas que recogía el anterior. La Delegación Nacional de Prensa, Propaganda y Radio del Movimiento, indica:

Es el órgano que, bajo la directa dependencia del Ministro Secretario General, tiene a su cargo la dirección política y la orientación de la propaganda, así como la gestión administrativa y económica de todas las unidades de producción de Prensa, Radio y Publicaciones del Movimiento ${ }^{6}$.

El acento puesto en la gestión económico-administrativa busca asegurar la coordinación entre las distintas unidades de la prensa y de la radio en los aspectos político, técnico y económico, según criterio de máxima eficacia funcional. No es difícil deducir, como lo hace Montabes Pereira,

que un nuevo espíritu empresarial invade a la Cadena, aunque sólo sea a efectos de obtener unos mejores resultados políticos.?

Los objetivos publicitarios marcados por la Delegación para los periódicos y revistas era como mínimo de un 33,33 por ciento de la mancha de las publicaciones. Esta cifra queda muy lejos del 20 por ciento como máximo, que se imponía en los años cuarenta.

En estos momentos interesa que los periódicos sean rentables, y para ello se intentará incrementar las ventas de ejemplares y rentabilizarlos con publicidad. De aquí que se cree en 1959 el término «Rendimiento Económico»" que es el tanto por ciento que representan los ingresos por publicidad con respecto al total de ingresos por todos los conceptos y tantos por ciento del total general de gastos que cubren dichos ingresos.

El estudio del rendimiento económico de la publicidad de las publicaciones de la Cadena, se hacía partiendo de la premisa de que los ingresos que se obtenían por la publicidad (y nos referimos a los ingresos brutos que son las cifras con las que se trabajaba), debían cubrir totalmente los gastos que producía la explotación del periódico, semanario o revista, como mínimo, quedando los ingresos que se obtuviesen por venta en plaza, corresponsales, suscriptores y varios como beneficios.

Cuando estos resultados no se obtenían había que pensar que en la publicación que se tratase se daba alguna de las circunstancias siguientes, que habría que rectificar?:

6 ZALBIDEA BENGOA, B. (1996), Op. cit, p. 46

7 MONTABES PEREIRA, J. (1989) La prensa del estado durante la transición política española, Centro de Investigaciones Sociológicas, Madrid, p. 31.

8 El término Rendimiento Económico se define y comienza a utilizar en el informe estudio sobre la publicidad insertada en los diarios y revistas de la Prensa del Movimiento, durante el ejercicio de 1958, comparada con la de los ejercicios económicos de 1956 y 1957 y porcentajes económicos que representa la misma. Fuente: Archivo General de la Administración. Sección Cultura. (1959) Signatura 12.

9 ARCHIVO GENERAL DE LA ADMINISTRACIÓN. Sección Cultura. (1959). Informe estudio sobre la publicidad insertada en los diarios y revistas de la Prensa del Movimiento, durante el ejerci- 
Que la publicación se halla recargada de gastos.

Que por causas de la tirada, situación geográfica y competencia, es un periódico pobre de publicidad.

Que su tirada no se encuentra al nivel que debiera, teniendo en cuenta las circunstancias citadas en el apartado anterior.

Si tomamos como herramienta básica el índice de rendimiento económico, la disminución del porcentaje de publicidad insertada se atribuirá a dos causas:

Aumento de páginas editadas sin que lo justificara la demanda de inserción de publicidad.

Un mayor porcentaje de gastos generales.

La realidad era bien diferente, los diarios no llegaban al 33,33\% de inserción publicitaria, lo cierto era que sus índices publicitarios, aunque iban en aumento, estaban muy lejos de conseguir esas cifras, pues los promedios estaban en 1958 en 19,90\%. Aunque superior al de los años 1956 y 1957 (16,96\% y 16,58\%) todavía muy lejos del objetivo fijado.

Estos nuevos objetivos publicitarios de la Prensa del Movimiento permitirán que en un futuro se crease lo que podríamos denominar el departamento comercial.

Con la década de los sesenta comienzan los años de bonanza para la Prensa del Movimiento, la difusión de los diarios continua en aumento, la rentabilidad de la Cadena crece hasta el 1968 y en el caso de los ingresos publicitarios se alcanzaron cifras espectaculares. Se pasó de unos ingresos líquidos de 146.802.007 ptas. a 577.856.299 ptas. en un período de diez años.

El auge publicitario de esta década y la creencia en la publicidad por parte de los dirigentes de la Cadena, hará de estos años los años dorados de la Prensa del Movimiento.

\section{La División Comercial}

El Decreto 2485/70, de 21 de agosto, por el que se dictaron las normas de organización y desarrollo de los Órganos de la Secretaria General del Movimiento, dedica el título VIII (artículos 54 al 60) a la Delegación Nacional de Prensa y Radio, y en su texto se advierte una importante laguna, la de no contemplar en ningún momento el hecho de que los medios de comunicación dependientes de la Delegación Nacional de Prensa y Radio del Movimiento son susceptibles de comercialización. En el

cio de 1958, comparada con la de los ejercicios económicos de 1956 y 1957 y porcentajes económicos que representa la misma. Signatura 12. 
citado Decreto no existe ninguna referencia a la acción comercial de dichos medios, aunque estos solamente contasen para subsistir con los ingresos que pudiera aportar la mencionada acción comercial.

En el proyecto de Norma Orgánica de la Delegación Nacional se contemplaba, sin embargo, la necesidad de una División Comercial encuadrada en el Departamento Económico-Administrativo.

La División Comercial se organizaba en tres vertientes de trabajo que correspondían a las tareas de distribución y venta de periódicos, promoción y venta de publicidad en la Cadena de Prensa y Radio, y venta de residuos industriales, todo ello según el criterio del Departamento Económico-Administrativo de la Delegación.

La función genérica de la División Comercial era aportar ingresos mediante la venta de sus productos, es decir, los periódicos, la publicidad y los residuos.

El Departamento de Publicidad de un periódico debía realizar las siguientes actividades, según el informe sobre funciones y estructuras de la Dirección Comercial ${ }^{1}$ :

- Promoción de cuentas y venta de espacios.

- Confección de originales o publirreportajes, cuando sea necesario, y colocación de la publicidad.

- Recepción y control de órdenes y originales.

- Servicio de ficheros y lanzamiento de promociones.

- Servicio técnico, de investigación y planeamiento de promociones.

- Relaciones públicas del medio.

El planteamiento de una acción publicitaria conjunta ayudaría a configurar la imagen de un periódico nacional, que es la propia Cadena de Prensa y Radio del Movimiento, compuesto fundamentalmente por 40 diarios con un ámbito de distribución y venta bien definido en cada caso, lo cual permitirá articular operaciones de venta de publicidad nacional por el mismo sistema utilizado por las revistas, pero con la ventaja de una garantía de distribución diaria y simultánea en todo el país.

Según los responsables de la Dirección Comercial una acción coordinada de ámbito nacional a través de los medios de prensa y de radio, debía contribuir a que la inversión publicitaria en España se canalizará de acuerdo con la política económica nacional, de forma que la publicidad no alcanzase un crecimiento superior a la renta nacional y que su inversión se realizara en los sectores adecuados.

A través del Departamento Económico-Administrativo, se mantendrían contactos con los Departamentos de Prensa y Radio para facilitarles las informaciones que pudieran resultarles útiles para el cometido de su competencia.

10 ARCHIVO GENERAL DE LA ADMINISTRACIÓN. Sección Cultura. (1971). Informe sobre Funciones y Estructura de la División Comercial. Signatura 5. 
"Se estudiará la posibilidad de mejorar la impresión en determinados periódicos, agilizar la información que resulte conveniente, realizar determinados números extraordinarios, llevar a cabo concursos y promociones facilitando los premios mediante intercambios de publicidad con las firmas que resulten adecuadas" ${ }^{2}$.

El Jefe de Publicidad de cada periódico recibía información de lo conseguido por la División Comercial, lo que le permitía llevar un cuadro estadístico de los objetivos conseguidos mediante las previsiones marcadas en el cuadro general, pidiendo a los Servicios Centrales cuanto necesitase para el mejor cumplimiento de estos objetivos. Disponía, asimismo, de la persona o personas precisas, cuando se estime que ello resultara razonablemente rentable; estudiaba y proponía cuantas acciones consideraba oportunas para conseguir sus objetivos; promocionaba los números extraordinarios, visitaba periódicamente a las Agencias y Anunciantes de su zona y se encargaba de supervisar la situación de los anuncios durante la confección del periódico, colaborando para ello con la persona o personas que designase el Director.

Todos y cada uno de los Jefes de Publicidad de los periódicos y emisoras debían recordar continuamente que eran parte de una gran Cadena y estarían dispuestos en todo momento a colaborar en las acciones comunes de ésta, tratando de crear entre sus Agencias y clientes la imagen de conjunto de la Cadena, para lo cual, recibían periódicamente información y material de la División Comercial.

Como consecuencia de todo lo anterior, se produjo un incremento en la realización de números extraordinarios de forma conjunta por todos los periódicos de la Cadena o por grupos de ellos.

Finalmente, las relaciones públicas al servicio de la acción comercial contaban con amplias posibilidades y funciones. Se trataba de estimular y mantener la confianza en la Cadena y en sus representantes por parte de los grupos humanos integrados en cada uno de los sectores afectados por la actuación de la Prensa del Movimiento.

Era absolutamente preciso desarrollar una conciencia comercial y una corriente de simpatía y confianza en el servicio que brindaba Prensa y Radio del Movimiento. La misión principal de la acción de relaciones públicas era establecer un nexo permanente de simpatía entre la División Comercial y las personas con quienes se relacionaba,

no en el escenario de la actividad comercial del negocio, sino en el del contacto humano, en los actos sociales, conferencias y coloquios sobre temas de publicidad, en la presencia de Prensa y Radio del Movimiento en ferias, exposiciones y certámenes seleccionados, mediante la creación de un "stand" con módulos adecuados a este fin. Hay que hacer las cosas bien y hacerlo saber. Por conducto de la acción de relaciones públicas tenemos que granjearnos en el mundo de las Agencias y Anunciantes el prestigio que merecemos. Hay que lograr también proyectar hacia el lector y hacia el oyente, hacia la sociedad en la que vivimos, una buena "imagen" de marca3.

11 A.G.A. Sección Cultura. (1971). Op cit.

12 A.G.A. Sección Cultura (1971). Informe sobre Funciones y Estructura de la División Comercial. Signatura 5. 
Al frente de la División Comercial se encontraba Ramos Perera Molina, que empezó a prestar sus servicios con fecha 12 de mayo de año 1971.

\section{El anuncio nacional.}

Al analizar las cifras publicitarias (1970-1971) de las publicaciones, en varias de ellas se puede observar que, mientras la publicidad de carácter local y regional se incrementaba, las cifras de la publicidad nacional sufrían un claro retroceso y, por otra parte, a la mayoría de los pequeños y medianos diarios no llegaban las campañas nacionales.

De aquí que fuese necesario poner en marcha los mecanismos para captar las citadas campañas, creando un anuncio conjunto para todos los diarios de la Cadena, que pudiese satisfacer a las Agencias, facilitándoles su labor al poner a su disposición un instrumento unificado con un mismo original y un precio bloque.

La determinación de este precio era un factor clave para la consecución de estos anuncios, y se imponía calcular un precio que, siendo sugestivo para las agencias, fuese beneficioso para la Cadena.

Otro aspecto que se estudió fue el tamaño del anuncio. Se pensó en tratar de encontrar unas medidas fijas que permitiesen con un solo original cubrir todos los diarios con un mínimo de pérdidas de espacio hasta completar los diferentes anchos de columna.

Los resultados publicitarios de la Cadena se incrementaban de forma positiva, pero este aumento se debía a la publicidad local o regional y, sobre todo, a un pequeño grupo de diarios que representaban el montante publicitario.

El sistema de Anuncio Nacional lograba convencer a las marcas de que, por un pequeño aumento en sus inversiones o una reducción en el tamaño de los anuncios, el anunciante conseguiría que su publicidad apareciera en todos los diarios. Con este planteamiento se conseguía el primer objetivo, que era la inserción de publicidad de marcas nacionales en todos los periódicos de la Cadena. De este modo, algunos dejaron de sentir la preocupante realidad de ver a estos mismos anunciantes en los diarios de la competencia, sin que a ellos le llegara ninguna página de estos grandes anunciantes, produciendo también en los anunciantes locales la consiguiente justificación para no acudir a esos periódicos porque cuando no iban las firmas nacionales sería porque ese diario no era interesante.

Ciertamente, el hecho de comparar los diarios de una misma provincia por su publicidad nacional, dio lugar a que se valorase aún más el Anuncio Nacional en la parte que podría influir en los anunciantes locales, pues consideraban que si un distribuidor local observaba que su marca decidía la inclusión de este periódico en su campaña nacional sería por razones de peso, al entender que la marca nacional tenía mayor formación publicitaria. 
La rentabilidad del Anuncio Nacional estaba asegurada desde el punto de vista social y su influencia local, pero su rentabilidad económica no era tal debido a los altos costes que se generaban con su realización y tramitación.

En el año 1972 se realizaron 105 Anuncios Nacionales, con un importe bruto de 24.308 .828 ptas. y en 1973 se realizaron 91 Anuncios Nacionales por un importe total de 19.840 .000 ptas., de éstos 196 anuncios publicados el beneficio final líquido ascendía a 809.284 ptas., lo que representaba un margen de beneficio del 1,9\%.

De lo anteriormente expuesto se llega a la conclusión de que a pesar del precio óptimo el anuncio nacional no logró un claro éxito, era preciso además de hacerlo rentable convertirlo en atractivo. Así, se planteó abrir un abanico de ofertas basadas, no tanto en los beneficios económicos, sino sobre todo en el servicio prestado.

Con los Anuncios en la Cadena Nacional se beneficiarían todas las campañas publicitarias, sea cual fuere su medida, formato, sección, fecha de inserción y emplazamiento, siempre que la orden fuera para un mismo anuncio y comprendiera toda la Cadena.

Con el objeto de reducir los costos de facturación de las campañas publicitarias de los anunciantes se crearon en la Cadena de Prensa del Movimiento una serie de "espacios tipo" ajustados a las siguientes condiciones de contratación. La compra de estos anuncios "tipo" estaba reservada de los miércoles a los sábados, ambos inclusive.

Asimismo, para facilitar también condiciones de contratación regional y con ello mayor facturación publicitaria, Prensa del Movimiento establecía la posibilidad de realizar campañas regionales, de acuerdo con el área geográfica mercadológica que en cada caso interesara.

En enero de 1977 la Cadena de Prensa del Movimiento editaría sus últimas tarifas con el slogan "Un anuncio para toda España...,... nuestro Anuncio Nacional" ", para un total de 31 periódicos de mañana y cuatro de tarde.

Por Real Decreto 1.501/77 de 2 de junio, a los efectos de lo previsto en la Ley General Presupuestaria, se clasificaba al Organismo Autónomo "Medios de Comunicación Social del Estado", con carácter "comercial, industrial, financiera o análogo".

Por Orden de 20 de junio de 1977 se dictaron una serie de normas complementarias al Decreto de 15 de abril, que desarrollaba el Organigrama del Organismo Autónomo y por el que se definían las funciones y estructura de la Dirección-Gerencia con los Departamentos de Prensa, Radio y Económico-Administrativo. Igualmente, se definían las Direcciones de los Medios Prensa y Radio, así como las Administraciones de dichos Medios.

13 A.G.A. Sección Cultura. (1977). Tarifas del Anuncio Nacional y Regional del año 1977. Signatura 21. 
Por Real Decreto 2258/77 de 27 de agosto, se daba estructura orgánica al Ministerio de Cultura y en su artículo $2^{\circ}$ punto $5^{\circ}$ se declaraba que el Organismo Autónomo "Medios de Comunicación Social del Estado" quedaba adscrito al Departamento Ministerial a través de la Subsecretaría del mismo.

Los MCSE se constituyeron con 35 diarios. Sus ingresos publicitarios, que fueron en aumento en la última década, ascendieron a mil cuatrocientos millones de pesetas para el año 1977.

La producción publicitaria de las publicaciones de la Prensa del Movimiento aumentó en un $418 \%$ en el período comprendido desde la Ley de Fraga hasta a la constitución de los MCSE (1966-1977).

A partir de estos momentos, aunque la inversión publicitaria continuaría en aumento, la mayor parte de los periódicos locales no son rentables, motivando el cierre de diarios. Siendo ésta una constante para una mayoría de ellos hasta la desaparición de los Medios de Comunicación Social del Estado.

\section{Conclusión}

Una vez analizado y reconstruido todo el proceso por el que pasa la publicidad en los diarios de la Cadena del Movimiento podemos destacar una primera etapa de postguerra donde la prensa es consideraba el principal motor propagandístico del régimen. La escasez de papel y de recursos hace que la publicidad quede en un segundo término pues es necesario aprovechar todo el papel para propagar las ideologías del Régimen y la poca publicidad que se inserta tiene un fuerte componente propagandístico. Con la entrada al gobierno de los tecnócratas en 1957 se abre una segunda etapa. En ella se le da más relevancia a la gestión administrativa y económica y se potencia un espíritu empresarial aunque sólo sea para conseguir unos mejores resultados políticos. La tercera etapa parte en el 1970 y en ella la Delegación Nacional de Prensa y Radio del Movimiento estructura y define las funciones de la Dirección Comercial de los diarios de la Cadena, buscando fórmulas y fomentando la comercialización de todos los periódicos en lo que se llamará el Anuncio Nacional. En este período existe una clara visión de rentabilizar cada uno de los diarios locales bajo el paraguas de la distribución nacional de la publicidad. En 1977 se da por finalizada la Prensa del Movimiento con la nueva denominación de Medios de Comunicación Social del Estado. 


\section{Bibliografía}

ARCHIVO GENERAL DE LA ADMINISTRACIÓN. Sección Cultura. Se han consultado todos los documentos y legajos en poder del AGA que hacen referencia a la Prensa del Movimiento.

7.1 Libros:

CIRICI PELLICER, A,(1977). La estética del franquismo, Gustavo Gilli, Barcelona. HERAS PEDROSA, C. de las (2000). La prensa del movimiento y su gestión publicitaria. SPICUM. Málaga.

MONTABES PEREIRA, J. (1989) La prensa del estado durante la transición política española, Centro de Investigaciones Sociológicas, Madrid.

PRADOS y LÓPEZ, M. (1943). Ética y estética del periodismo español. Espasa-Calpe. Madrid.

SINOVA, J. (1988). La censura de prensa durante el franquismo. Espasa-Calpe. Madrid.

TIMOTEO ÁLVAREZ, J. (1989). Historia de los medios de comunicación en España. Ariel. Barcelona.

ZALBIDEA BENGOA, B. (1996) Prensa del Movimiento en España. Universidad del País Vasco.

\section{Los autores}

Carlos de las Heras Pedrosa. Licenciado en Ciencias Económicas y Empresariales y Doctor en Publicidad y Relaciones Públicas por la Universidad de Málaga. Es profesor Titular de Universidad en el Área de Conocimiento de Comunicación Audiovisual y Publicidad. Actualmente imparte la docencia en la asignatura de Comunicación Institucional. Su vida investigadora ha girado en torno a dos grandes líneas: La historia de la publicidad y la prensa en España, la comunicación institucional y la comunicación turística. En esta última, profundiza aspectos relacionados con el patrocinio, la imagen de las instituciones y de sus líderes. Es autor de dos libros y coordinador de un tercero. Asimismo, cuenta con numerosas aportaciones en capítulos de libros, revistas y congresos nacionales e internacionales. Entre sus cargos académicos y de gestión destacan el de Codirector del Programa de Doctorado "Comunicación Organizacional"; Director del "Máster Universitario de Dirección en Comunicación y Turismo"; y Vicedecano de la Facultad de Ciencias de la Comunicación.Ha sido Subdirector General de Comunicación, Protocolo y Gabinete de la Rectora, posteriormente ejerció como Director del Gabinete del Rectorado (con rango de Vicerrector). Actualmente es Vicerrector de Relaciones Institucionales y Gabinete del Rectorado.

Carmen Jambrino Maldonado. Doctora en Ciencias Económicas y Empresariales. Catedrática de Escuela universitaria del Área de Comercialización e Investigación de 
Mercados. Directora del grupo de Investigación Marketing para Pymes" financiado por la Junta de Andalucía. Directora del X Master universitario de Marketing de Negocios de la Universidad de Málaga. Directora de programa de doctorado Investigación y Marketing. Ha participado en Congresos Nacionales e Internacionales y publicado artículos en diversas revistas científicas. Miembro de la Academia Europea de Economía y Dirección de Empresas y de la -Asociación científica de Economía y Dirección de empresas. Ha participado en contratos de especial relevancia con empresas y administraciones públicas como "Auditoría de Imagen de la Costa del Sol" para Turismo Andaluz, junta de Andalucía o Programa de Identidad Corporativa de la Asociación Internacional Club Biored. 